\title{
Transatlantica
}

Revue d'études américaines. American Studies Journal

\section{Révoltes, révolution, radicalisme}

Les concepts en usage dans la Révolution américaine et son

historiographie

\section{Elise Marienstras}

\section{(2) OpenEdition}

1 Journals

Édition électronique

URL : http://journals.openedition.org/transatlantica/1143

DOI : $10.4000 /$ transatlantica. 1143

ISSN : 1765-2766

Éditeur

AFEA

Référence électronique

Elise Marienstras, «Révoltes, révolution, radicalisme », Transatlantica [En ligne], 2 | 2006, mis en ligne le 07 juillet 2006, consulté le 29 avril 2021. URL : http://journals.openedition.org/transatlantica/1143 ; DOI : https://doi.org/10.4000/transatlantica. 1143

Ce document a été généré automatiquement le 29 avril 2021

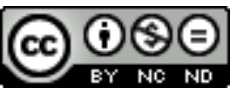

Transatlantica - Revue d'études américaines est mis à disposition selon les termes de la licence Creative Commons Attribution - Pas d'Utilisation Commerciale - Pas de Modification 4.0 International. 


\title{
Révoltes, révolution, radicalisme
}

\author{
Les concepts en usage dans la Révolution américaine et son \\ historiographie
}

Elise Marienstras

1 Il y a fort longtemps, j'ai passé le Capes une première fois, et, bien que reçue, je l'ai passé une deuxième fois parce que je n'avais pas fait le stage requis. Puis j'ai passé l'agrégation, deux fois aussi pour raisons de faiblesse en géographie. De tout cela ainsi que des différents examens et dissertations, thèses ou mémoires qu'un universitaire mène à bien (ou à moins bien) au long de sa vie, j'ai retenu une première leçon : il faut toujours flatter son jury, lui dire combien on a apprécié la formulation du sujet d'examen ainsi, d'ailleurs, que de la question mise au programme - et le choix des repères chronologiques, toujours arbitraires naturellement, mais bien venus. Vous ferez donc une analyse sémantique si possible enthousiaste (et érudite) de l'intitulé du sujet et de la question et ce faisant, vous serez peut-être sincères, en tout cas en matière d'histoire de la Révolution américaine. ${ }^{1}$

2 En effet, les dates indiquées (1773-1802) dépendent plutôt de la chronologie de la Révolution française que de l'américaine. Mais, comme Marcel Dorigny l'écrit si bien dans son ouvrage consacré à la question au programme, 1773 (année des Tea Parties à Boston, à New York et ailleurs) convient parfaitement pour dater, non pas tant, comme il le dit, le début de la Révolution américaine, que le moment «tournant»-celui qui fait l'articulation entre les deux concepts indiqués aussi dans l'intitulé de la question: révoltes et révolution (Dorigny 90-92).

3 L'année 1802 est peut-être moins significative quant à la Révolution américaine, mais elle fait aussi la jonction entre d'une part, la prétendue « révolution » qu'annonçait en 1800 le nouveau et troisième président des Etats-Unis, Thomas Jefferson, en promettant d'abroger les lois liberticides du gouvernement précédent, et d'autre part l'achat qu'il fit de la Louisiane à Bonaparte en 1803. Jefferson ne faisait alors que saisir l'occasion que lui offrait Bonaparte en difficulté. Mais en vouant de la sorte la nation à occuper tout un continent, il mettait le mot « fin » à la Révolution de 1776 qui avait arraché les treize colonies à l'empire britannique et en avait fait une république ; à partir de 1803, la jeune et petite nation qui s'était contentée de se rêver empire, allait pouvoir 
commencer à réaliser ce qu'on appellerait sa « mission » en fondant, selon l'expression de Jefferson, un « empire de la liberté ».

4 Terminer la question en 1802 ouvre donc, pour l'Amérique du Nord, la question de l'expansionnisme inhérent à la Révolution américaine, cependant que 1789, qui forme, à l'intérieur du sujet, une date charnière, marque le début convenu de la Révolution française, en même temps que, aux Etats-Unis, on assiste à l'aboutissement de la Révolution politique - la stabilisation du régime que vient d'inventer la Convention constitutionnelle. (En avril 1789, les deux Chambres législatives du Congrès se réunissent et élisent le premier président des Etats-Unis, George Washington). La Révolution américaine peut donc se targuer, vis-à-vis de la Révolution française à laquelle on la compare, du mérite de l'antériorité. C'est un fait dont les comparatistes tirent, selon leur orientation propre, des conclusions contradictoires. Les "exceptionnalistes" français ont longtemps ignoré la Révolution américaine, la jugeant imparfaite parce que non sociale; alors que les «exceptionnalistes" américains condamnaient la violence de la Révolution française et son échec final pour mettre en avant l'exemplarité et l'influence universelle et éternelle de la Révolution américaine.

5 Pendant longtemps, les historiens contemporains de la Révolution ou leurs successeurs $\mathrm{du} \mathrm{XIX}^{\mathrm{e}}$ siècle, dits "filio-piétistes" (par respect pour leurs pères whigs ou révolutionnaires; Ramsay, Bancroft), puis plus récemment ceux qui se disaient " whigs » et que l'on appelle par dérision « historiens du consensus » (Boorstin, Baylin) ont vu dans la Révolution américaine essentiellement la confrontation des colons et de la Couronne britannique, en matière fiduciaire, constitutionnelle, et militaire.

6 C'est cette confrontation et ses suites, c'est-à-dire l'adoption d'une république fédérale et d'une Déclaration des droits, qui sera considérée comme La Révolution américaine. Et qui sera présentée dans le réseau des "révolutions atlantiques ", par cette tradition historiographique, comme la révolution modèle, ou comme l'inspiratrice de celles qui l'ont suivie, ou encore et surtout comme l'exemple unique d'une révolution réussie (Morris, Arendt). De là s'est établie (et répandue dans le monde) la théorie ou la légende, de l'«exceptionnalisme américain » qui a encore souvent cours aujourd'hui. Ces historiens de la guerre froide, étaient, en premier lieu, fortement hostiles à d'autres révolutionnaires, les Bolcheviques et staliniens dont ils trouvaient les prédécesseurs chez les activistes français de la Terreur.

7 A l'inverse, la génération des historiens "radicaux", encore étudiants ou jeunes professeurs dans les années 1960, rapprochait ouvertement l'objet de ses recherches des préoccupations et des luttes de son temps. "Red diapers ${ }^{2}$ " pour certains, ils se voulaient d'abord historiens "sociaux» et étaient teintés de marxisme. Ils participèrent aux mouvements sociaux de leurs temps qui protestaient contre la guerre du Vietnam ainsi que contre les inégalités flagrantes entre la majorité de la population et les minorités défavorisées. Ces mêmes historiens, sans que leur discipline en subît de contre-coup sur le plan des méthodes et de la qualité s'investirent dans l'histoire des « radicaux » du passé, et voulurent à la fois voir l'histoire non plus depuis le point de vue de l'élite cultivée et agissante, mais, selon une formule célèbre « depuis le bas de la société vers le haut" (from the bottom up, Lemisch) et en restituant aux acteurs oubliés - petit peuple, femmes, noirs, indiens - leur place dans l'histoire.

De ce relativisme historiographique, on devra tirer une deuxième leçon: il est très important de ne pas « réifier» les concepts (ce serait d'ailleurs une impasse en forme 
d'oxymore), en en cherchant une définition unique et datée de manière précise, dans un seul dictionnaire. Ceux qui se contentent de cette source d'information et d'interprétation en croyant ainsi bien planter le sens d'un mot, d'un concept plutôt, dans son temps et éviter l'anachronisme, ignorent à quel point l'historien est une personne plongée dans son propre temps autant que dans les archives du temps passé.

Il est préférable de consulter plusieurs dictionnaires et encyclopédies historiques, sans parler naturellement des archives du xviII ${ }^{e}$ siècle, ce qui nous permettra de rendre vie aux concepts et de les contextualiser. Il est aussi très important de garder à l'esprit l'historiographie qui se rapporte aux concepts étudiés, puisque aussi bien l'époque que l'historien avec sa subjectivité et son rapport avec son temps, influent sur les mots et les idées, et par là, peuvent même infléchir le cours de l'histoire, si tant est que les idées jouent un rôle moteur dans le dynamisme historique, notamment dans le cours des révolutions.

On a compris que les concepts auxquels je faisais allusion - les 3 « $R$ » (non pas Reading, Writing et Reckoning) - , mais "Révoltes, Révolution et Radicalisme", et encore "Résistance ", "Rébellions ", "Réforme " ainsi que "Sédition » ou "Insurrections " sont des mots à double face, c'est-à-dire qu'ils peuvent être précédés d'un signe positif ou négatif, selon les acteurs et les observateurs. A cette ambivalence générale, il faut ajouter, pour l'Amérique du Nord britannique, la complexité et les particularités du conflit entre les colonies britanniques et leur métropole depuis les années 1760 .

11 Car si la révolution américaine présente des traits communs à toutes les révolutions de l'ère moderne et contemporaine - une conception du temps hors norme, une densité du phénomène révolutionnaire ainsi que l'absence de causalité linéaire due à la complexité, la multiplicité, l'aléatoire même des causes à l'origine de la révolution-(Marienstras 21-23), elle diffère en tout cas clairement des autres révolutions par certains aspects notables :

On y constate en effet que résistance, révoltes et révolutions s'y font, si l'on peut dire, "à l'endroit et à l'envers ", vers l'extérieur comme de manière intestine, contre des frères ennemis, les "loyalistes", aussi bien que contre des envahisseurs et des oppresseurs. Ceux qui résistent sont souvent aussi ceux qui oppriment. La résistance des uns sera victorieuse et conduira à une plus grande oppression des autres. En nous attardant sur cet aspect duel, on voit que les concepts principaux en usage au temps de la révolution, et tels qu'ils sont présentés par les divers historiens sont revêtus d'un signe particulier que leur confère le phénomène colonial. Les concepts de "résistance», celui de "révoltes», de "réformes», de «radicalisme» et de "révolution » n'ont pas le même sens dans un espace de colonisation qu'à l'intérieur d'un Etat-nation déjà constitué. Il est alors intéressant de se livrer au comparatisme avec la Révolution en France ou en Haïti, sans parler des nombreuses résistances, révoltes et rébellions dans le monde occidental et centre et est-européen du $\mathrm{XVIII}^{\mathrm{e}}$ siècle.

13 Voyons donc l'Amérique des colons britanniques en 1765, date à laquelle commence le malentendu entre l'Angleterre et ses colons. Par la suite, l'innovation formidable qui résultera des événements américano-anglais de la fin du XVIII ${ }^{\mathrm{e}}$ siècle se produira de manière presque impromptue, sous la force, quoiqu'on en ait souvent dit, d'un enchaînement de circonstances largement improvisées (notons au passage que le mot révolution prend ici le même sens que l'émeute qui n'est pas plus préméditée. Notons aussi que j'avance ici une interprétation de la révolution contraire à celle de John 
Adams, par exemple, qui dira quelque vingt ans plus tard, que la « révolution était déjà dans les têtes avant d'être dans les cœurs ", Adams).

14 La première étape de la Révolution américaine, qui aurait pu s'arrêter là sans l'obstination de la Grande-Bretagne à réformer l'empire pour y assurer sa suprématie (les colons aussi voulaient le réformer, mais dans le sens inverse), fut une résistance et finalement une révolte contre le système mercantiliste. Commencée en 1763 comme un conflit fiscal en partie comparable à ceux qui suscitèrent ailleurs les grandes révoltes du siècle, la résistance des colons tourna à l'insurrection parce que cette fiscalité s'exerçait à l'intérieur d'un empire dont ils étaient les sujets éloignés. Ainsi peut-on mettre en exergue le caractère anti-colonial de la Révolution américaine, motivée en premier lieu par des considérations économiques, auxquelles, en second lieu, viendra se greffer une rhétorique inspirée par de fortes motivations politiques. On voit dès lors que le processus est fort différent de celui de la France révolutionnaire, et encore plus de celui de Haïti. La révolution à Haïti est elle aussi anticoloniale, mais elle est faite par les esclaves, frères en servitude de ceux qui, dans les colonies britanniques du sud des Etats-Unis, allaient pour la plupart pâtir des résultats de la révolution à la fois coloniale et anticoloniale qu'avaient menée leurs maîtres.

Quelle est la différence entre la résistance et la révolte ? La première est parée par nos contemporains français du noble signe de l'héroïsme et du patriotisme. Dans les colonies britanniques en cette fin du XVIII ${ }^{e}$ siècle, elle dénote l'action souvent passive (on résiste le dos au mur, on résiste à la poussée, à la violence) de personnes qui au nom d'une juste cause, celle de la liberté des sujets anglais, refusent de se plier à la violence ou à la pression qui s'exercent contre eux. Il s'agit d'abord des colons (riches marchands comme John Hancock, notables comme les cousins Samuel et John Adams, avocats en Nouvelle-Angleterre, Patrick Henry et tant de riches planteurs et avocats de Virginie...) qui résistent aux lois votées à l'encontre, disent-ils, de leur droit à commercer librement et à ne "pas être taxés sans leur consentement». Cette résistance tourne à la rébellion lorsque la Couronne veut imposer une taxe intérieure sur le Timbre, au mépris de l'autonomie des assemblées coloniales. La rébellion est alors assimilée par le roi d'Angleterre à un début d'insurrection. Les premiers résistants se muèrent en acteurs déterminés à faire valoir leurs droits, y compris par la force. Il y eut attaque de percepteurs du Timbre, formation d'organisations (le Congrès du Stamp Act) destinées à rallier toutes les colonies, d'associations s'en prenant directement aux moyens du commerce britannique. C'est pourtant par le but visé, que l'on peut dire que cette première rébellion ne fut pas une révolution : avant 1774,1775 ou même encore 1776, tous les rebelles n'étaient pas décidés à renverser le régime politique sous lequel ils vivaient et à lui substituer une république indépendante.

Il était cependant question depuis longtemps, dans les classes populaires, d'un mécontentement, d'une inquiétude, d'une colère, qui provoquèrent, avant même l'éclatement du conflit impérial, des révoltes agraires, des émeutes urbaines, des rébellions de matelots contre l'enrôlement forcé dans la marine britannique. Il s'agit d'une forme de résistance qui précéda, et accompagna la résistance au mercantilisme, s'emboîtant dans le mouvement des notables, y prêtant main forte lorsqu'il apparaissait qu'ils avaient le même oppresseur, mais se faisant à l'inverse loyaliste lorsque les colons «patriotes » étaient en même temps les députés aux assemblées qui votaient des impôts excessifs et menaçaient d'expulser les squatters de l'intérieur, ou les bailleurs abusifs des métairies, ou les planteurs riches de terres qui employaient les 
serviteurs engagés, ou les juges des tribunaux de comtés qui emprisonnaient le pauvre monde pour dettes ou non paiement d'impôts. On trouve ici une conjonction singulière de revendications de classe, si l'on peut dire, qui unissent sous un même drapeau les sans-terre misérables des campagnes (environ $40 \%$ de la population) et les apprentis des villes, sans espoir de devenir artisans faute d'un petit capital, aux fermiers bien lotis mais chargés d'impôts ainsi qu'aux esprits avides d'une religiosité sans fard et sans hiérarchie.

Jusqu'à quel point ces revendications disparates étaient-elles empreintes d'une même idéologie démocratique révolutionnaire qui aurait précédé de loin la révolution menée par l'élite contre la monarchie anglaise? Question difficile à trancher et qui fait pourtant la thèse des historiens radicaux - Alfred Young (qui édita deux volumes d'historiens «radicaux» sur le radicalisme de la Révolution, 1976, 1993), Edward Countryman, Eric Foner, Jesse Lemisch, Markus Rediker et bien d'autres. Il existe quelques textes qui soutiennent par exemple, comme l'ouvrage de William Manning, un fermier et tenancier de taverne, qui écrivit en anticipation du mutualisme des travailleurs, que "le travail est l'unique source de la propriété », et que les fermiers " ont droit au fruit de leur labeur produit à la sueur de leur front» (Manning). "Les fermiers qui labouraient un sillon, semaient les graines et récoltaient la moisson, comme les fermières qui filaient, tissaient, puis cousaient les vêtements n'avaient pas besoin de leçons d'économie politique, pour apprendre la théorie de la plus-value créée par le travail » écrit Al Young (1993). C'est l'expérience de la précarité que leur donnait leur condition qui faisait craindre aux travailleurs, même un peu dotés, de perdre le peu de sécurité dont ils disposaient. Et si la rhétorique politique et constitutionnelle des dirigeants « whigs » ou " libéraux » rencontrait un écho parmi eux, c'est parce que cette rhétorique traduisait, d'une certaine manière, les préoccupations et la colère du peuple contre les oligarques dont ils dépendaient.

18 Mais la rhétorique «libérale» révolutionnaire n'en était pas pour cela radicale. Les structures de la société furent peu modifiées par la Révolution: quelques terres des émigrés loyalistes furent distribuées à des anciens combattants; quelques esclaves furent affranchis parce qu'ils avaient combattu avec - mais pas aux côtés - de leurs maîtres « patriotes "; la Révolution signa aussi, dit Gordon Wood, la fin de la "société de déférence ", celle où les humbles enlevaient leur chapeau devant les propriétaires et élisaient ceux-ci régulièrement pour les représenter. Gordon Wood semble prendre une certaine égalité de manières et le refus de l'ostentation chez les riches, pour l'avènement de l'égalité des conditions, alors que le fossé se creuse entre les libres et les esclaves, entre les autochtones et les Euro-Américains, entre les grands marchands et les pauvres artisans, bientôt entre les entrepreneurs et les ouvriers. Surtout, l'égalité des droits a été proclamée et inscrite dans la Déclaration d'indépendance, puis dans les dix premiers amendements à la Constitution; il ne restait qu'à la donner aux femmes, aux Noirs, aux Indiens et aux apprentis, et à commencer à l'appliquer, ce qui prendrait encore maintes décennies, et même, peut-on dire, au moins deux siècles à parfaire.

19 La question reste posée: qu'est-ce et qu'était-ce qu'un radical, qu'est-ce que le radicalisme? Evitons de donner une définition, puisque le « radical » John Adams, par exemple, dénonça les émeutiers vulgaires et violents lorsqu'il plaida pour les officiers britanniques après le massacre de Boston; que Thomas Jefferson, qui déplorait qu'un jour le péché originel des Américains - l'esclavage - n'entraînât une sanglante guerre civile, avait lui-même une centaine d'esclaves qu'il faisait rarement fouetter; que 
Robert Morris, financier de la Révolution et signataire de tous les documents fondateurs américains, craignait par dessus tout, lors de la révolte agraire de Shays, en 1787, que le régime confédéral ne produise l'anarchie et la destruction de la propriété ; et que le véritable radical que fut Thomas Paine, mourut pauvre et répudié par les bénéficiaires de la Révolution américaine!

Ainsi, alors que les historiens dits "whigs» ou filio-piétistes, ainsi que ceux « du consensus », collent l'étiquette de " radicaux » à des révolutionnaires, tous membres de l'élite, comme John et Samuel Adams ou Patrick Henry, parce qu'ils sont allés jusqu'au bout de la rupture avec la monarchie anglaise et ont proclamé les droits de l'homme, les historiens radicaux nomment ces derniers des "libéraux », et, en ce qui concerne certains révolutionnaires comme John Jay ou Alexander Hamilton, les qualifient de " conservateurs » parce qu'ils se sont méfiés du « despotisme de la majorité », (c'est-àdire de la démocratie) et ont instauré en 1787 un régime fédéraliste «ni national ni fédéral » ou les deux à la fois, pourvu que la propriété, y compris en personnes humaines, soit préservée.

Qui fut donc radical pendant la Révolution américaine, selon les historiens radicaux dont Al Young fait une synthèse interprétative? C'est en fin de compte pour lui la majorité des colons, ces petits fermiers, ces pauvres, ces squatters, ces apprentisartisans, ces marins, ces femmes, que la conscience d'être opprimés fait, selon les historiens radicaux, des radicaux eux-mêmes. Ils porteront leurs revendications audelà de la Révolution, en s'opposant au fédéralisme dont ils sentent bien qu'il leur confisquera le pouvoir et méconnaîtra les véritables droits du peuple.

Mais eux-mêmes, ces travailleurs immigrés ou descendants d'immigrés, quel fut leur œuvre dans la Révolution? Une certaine confusion règne entre la rhétorique de leurs porte-paroles et les actions menées - rébellions contre les tribunaux, émeutes contre les soldats anglais, prise de pouvoir parfois comme au Vermont où Ethan Allen mène l'occupation des terres des spéculateurs. Quels en furent les résultats, le poids sur l'effet social de la Révolution? Al Young nous dit que, grâce à la pression exercée par les Antifédéralistes dont la composition sociale n'est pas claire, la Constitution de 1787 fut plus libérale, plus démocratique (encore un concept à décrypter) que l'on ne s'y attendait (Young 1988). Elle resta cependant ce qu'avaient eu l'intention d'en faire les planteurs sudistes et leurs alliés du nord: esclavagiste et exclusive. Les Africains y furent traités comme des "personnes » objets de propriété et marchandises en même temps qu'outils de production. Les Amérindiens reçurent un statut ambigu, mais hors de la république. Les femmes ne reçurent aucun statut autre que mineur et donc dépendant. C'est, à mes yeux, la prise de conscience et la lutte, par les Amérindiens et plus tard par les Noirs, contre le caractère colonial des Etats-Unis qui est au cœur du radicalisme.

Par ailleurs, le radicalisme reste, pour nous d'aujourd'hui, une notion molle, comme on dit « une science molle » pour les sciences humaines. Les Américains pour lesquels les termes de gauche et de droite ne sont pas idiomatiques tentent de rapprocher le concept de «radicalisme» de celui de "nouvelle gauche » qui fut en usage dans les années 1960 et eut alors un sens précis. Mais est-il possible d'aller aussi loin que le fait Eric Foner en faisant des deux notions des synonymes de socialisme? (Foner 1998) On est dès lors bien loin de la Révolution américaine du XvIII ${ }^{e}$ siècle. 


\section{BIBLIOGRAPHIE}

Adams, John to Hezekiah Niles. February 13, 1818. The Works of John Adams. Ed. Charles Francis Adams. 10 vol. Boston, 1856.

Arendt, Hannah. Essai sur la Révolution (1963). Tr. fr. Paris : Gallimard, 1985.

Bancroft, George. History of the American Revolution. 7 vols. Boston : Little Brown \& Company, 1852-74.

Countryman, Edward. The American Revolution. New York : Hill and Wang, 1985.

Dorigny, Marcel, ed. Révoltes et révolutions en Europe et aux Amériques (1773-1802). Paris : Belin, 2004.

Foner, Eric. Tom Paine and Revolutionary America. New York : OUP, 1976.

Foner, Eric. « Common Origins, Different Paths ». Forum on Liberalism and the Left : Rethinking the Relationship. Radical History Review 71 (1998 b). http://2act.org/metaPage/lib/The-Leftagainst-liberalism.pdf.

Lemisch, Jesse. «The American Revolution Seen from the Bottom Up ». Towards a New Past : Dissenting Essays in American History. Ed. Barton J. Bernstein. New York : Knopf, 1968, 3-45.

Maier, Pauline. From Resistance to Revolution. Colonial Radicals and the Development of American Opposition to Britain, 1765-1776 (1972). New York : Vintage, 1974.

Manning, William. The Key of Liberty. 1799. Ed. Michael Merrill \& Sean Wilentz. Cambridge, Mass. : Harvard University Press, 1993.

Marienstras, Elise. «La Révolution américaine : une création nationale, 1763-1800 ». Révoltes et Révolutions en Europe (Russie comprise) et aux Amériques de 1773 à 1802. Ed. Raymonde Monnier. Paris : Ellipses, 2004. 21-60.

Morgan, Edmund. « Revisions in Need of Revising, The Challenge of the American Revolution ». New York : Norton, 1976.

Morris Richard B. The Emerging Nations and the American Revolution. New York : Harper, 1970.

Paine, Thomas. Le Sens Commun. Traduction de Bernard Vincent. Paris : Aubier, 1983.

Ramsay, David. The History of the American Revolution. 2 vols. Philadelphie, 1789.

Rediker, Marcus. Between the Devil and the Deep Blue Sea : Merchant Seamen, Pirates, and the AngloAmerican Maritime World, 1700-1750. New York : Cambridge University Press, 1987.

Wood, Gordon. The Radicalism of the American Revolution. New York : Vintage, 1991.

Young, Alfred. F., ed. The American Revolution. De Kalb : Northern Illinois University Press, 1976.

Young, Alfred F., ed. Beyond the American Revolution. Explorations in the History of American Radicalism. De Kalb : Northern Illinois University Press, 1993.

Young, Alfred F. "The Framers of the Constitution and the 'Genius' of the People ». Beyond Beard: A Symposium. Radical History Review 42 (Fall 1988) : 8-18. 


\section{NOTES}

1. La question au programme de l'agrégation d'histoire en 2004 et 2005 était : « Révoltes et révolutions en Europe (Russie incluse) et aux Amériques de 1773 à 1802 ».

2. L'expression " red diapers » (littéralement « couches rouges », c'est-à-dire langés dans l'idéologie communiste de leurs parents) est employée avec fierté par le milieu d'intellectuels « radicaux ", actifs depuis la guerre du Vietnam et les combats pour les droits civiques, et dont les parents avaient appartenu à une gauche militante dans les années $1920-1930$ et 1950.

\section{AUTEUR}

\section{ELISE MARIENSTRAS}

Paris 7 